\title{
A Prolapsed Intervertbral Disc as the Presenting Feature for Alkaptouria: Case Report
}

\author{
Abdulrahman Al-Shudifat ${ }^{1,2^{*}}$, Khalid Alkharazi ${ }^{2}$, Qussay Salih Alsabbagh ${ }^{2}$ and Nosaiba T Ryalat ${ }^{3}$ \\ ${ }^{1}$ Department of Neurosurgery, Lund University, Sweden \\ ${ }^{2}$ Department of Neurosurgery, The University of Jordan, Jordan \\ ${ }^{3}$ Department of Radiology, The University of Jordan, Jordan
}

"Corresponding author: Abdulrahman Al-Shudifat, Department of Neurosurgery, Lund University, Sweden Tel: +46760807915; E-mail: abdulrahman.alshudifat@med.lu.se

Received date: January 25, 2016; Accepted date: February 18, 2016; Published date: February 22, 2016

Copyright: (c) 2016 Al-Shudifat A, et al. This is an open-access article distributed under the terms of the Creative Commons Attribution License, which permits unrestricted use, distribution, and reproduction in any medium, provided the original author and source are credited.

\begin{abstract}
Alkaptonuria is a rare metabolic disease caused by deficiency of homogentisic acid oxidase and characterized by bluish-black discoloration of cartilages and skin (ochronosis). Discal herniation requiring surgery is unusual in alkaptonuria, with only a few reports. The authors report the case of an elderly patient with lumbar disc herniation who underwent discectomy and in whom the nucleus pulposus was discovered to be black. Alkaptonuria was diagnosed after discectomy. The symptoms in the patient disappeared after surgery.
\end{abstract}

Keywords: Intervertbral disc; Alkaptouria; Chromosome; Neurological; Surgery

\section{Introduction}

Alkaptonuria, a rare autosomal recessive disorder of phenylalanine and tyrosine metabolism caused by deficiency in homogentisate 1,2 dioxygenase activity due to a chromosomal defect in the long arm of Chromosome 3 [1]. This leads to accumulation of large amounts of homogentisic acid. The oxidation and the polymerization of homogentisic acid leads to black coloration of standing urine and all connective tissues where it is deposited [2,3]. Pigmentation may be seen on the skin, the teeth, the nails, and the patient's buccal mucosa; this condition is called ochronosis [4,5]. Homogentisic acid is deposited as an oxidized and polymerised pigment (ochronotic pigment) in various tissues and organs binding irreversibly to collagen and causing bluish-black pigmentation (ochronosis). The ochronotic arthropathy affecting mainly the vertebral discs and large joints. The spinal involvement results in kyphosis, height loss, and decreased lumbar flexion, and decreases the range of motion and causes effusions [1]. Alkaptonuria most prominently involves the lumbar region, but lumbar disc herniation as the presenting feature of alkaptonuria is not common. The authors report a case of an elderly patient who underwent discectomy for a prolapsed lumbar disc when a black colored nucleus pulposus was retrieved. Alkaptonuria was diagnosed after discectomy. The clinical presentation, radiological and pathological findings are described.

\section{Case Report}

A sixty-five years old male patient, who's known to have hypertension, was admitted to the neurosurgical department with chronic low back pain that was exacerbated in the last year prior to his presentation, associated with left lower limb sciatica, numbness in the lateral aspect of the left foot and a history of progressive claudication.
Systemic review revealed multiple joints pains mostly in his hands, right shoulder and knees. Interestingly the patient reported passing dark colored urine since early childhood that was not worthing investigation according to him.

Examination of the skin revealed abnormal dark-bluish discoloration most evident over the ear pinna and the dorsum of both hands, (Figure 1). He showed limitation of flexion and extension in the thoracic and lumbar spines.

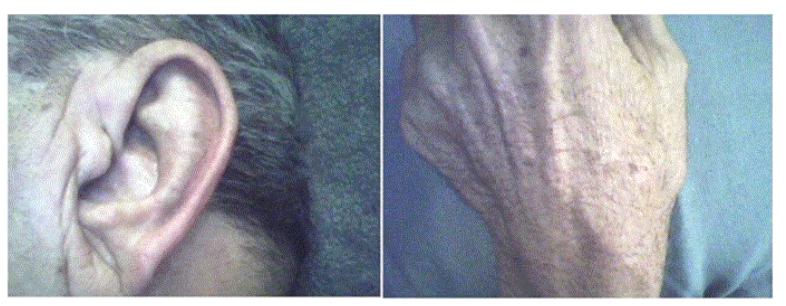

Figure 1: Abnormal bluish discoloration seen over the skin in the pinna of the ear and dorsum of the head.

Neurological evaluation was unremarkable except for hypoesthesia in the territory of left first sacral root and absent ankle jerk on the same side.

Dorsal and lumbar spine radiography showed narrowing of the disc spaces with calcification of the discs themselves, (Figure 2), and lumbar spine magnetic resonance imaging confirmed the presence of the degenerative changes but interestingly there were abnormal bone marrow signals (hyper intense on T1WI and on T2WI) in almost all the lumbar spine which were suppressed with fat sat study in addition to an up migrated prolapsed intervertebral disc on the left side of disc space between the fifth lumbar and the first sacral vertebrae, (Figure 3). 

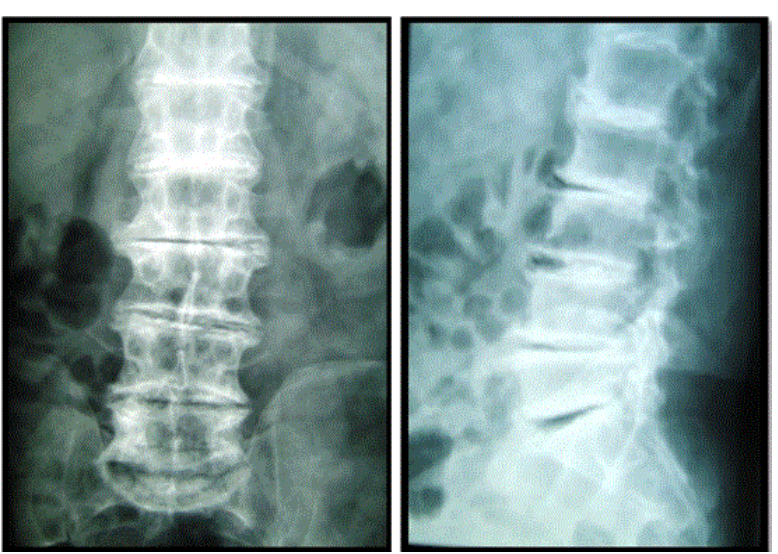

Figure 2: A lumbosacral spine X-Ray with severe degenerative changes and calcification of disc spaces.

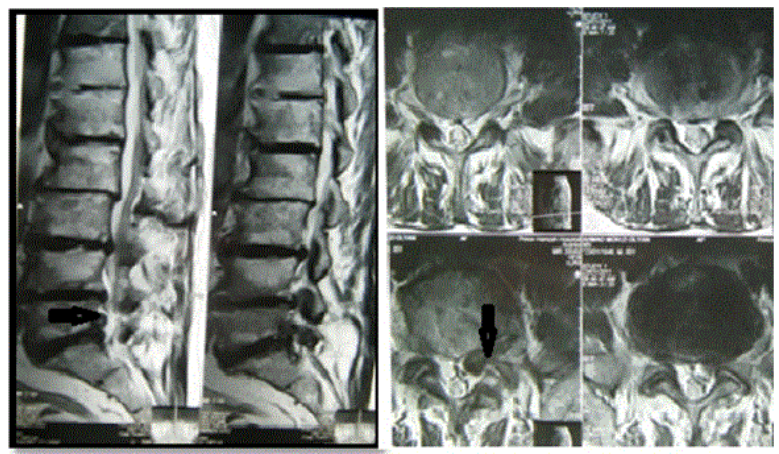

Figure 3: The magnatic resonance images of the lumbosavral spine showing the degenerative changes and the abnormal bone marrow signals in almost all the lumbar spine which were suppressed with fat sat study in addition to an up migrated prolapsed invertebral disc on the left side of the disc space between the fifth lumbar and first sacral vertebrae (arrow).

Surgery for the excision of the L5S1 prolapsed disc was performed using the stain H\&E in the routine technique by micro fenestration and microdiscectomy, but interestingly the bone and the ligamentum flavum which were removed had blackish blue colour, the prolapsed nucleus pulposus pieces had the same discoloration and all were send for the histopathological examination, (Figure 4).

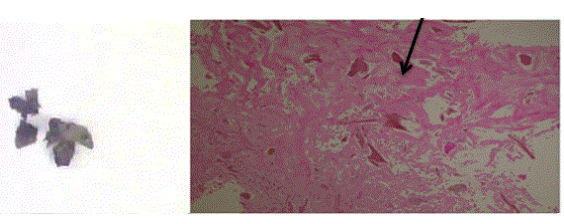

Figure 4: Left, the excised nucleus pulposus with the abnormal black discolouration. Right, the microscopic appearance of multiple pigmented areas deposits within the nucleus material and the pink ones are pigmented deposits.
Histopathological examination of the ligaments, bone and the nucleus pulposus showed the classic findings of ochronosis, including multiple pigmented areas deposits within the nucleus material, (Figure 4).

\section{Discussion}

$\mathrm{AKU}$ is a rare autosomal recessive condition with an incidence of $0.001 \%$, which has no effective treatment or cure [6]. The clinical manifestations and advanced ochronosis are well documented. The disease was discovered in Egyptian mummies in 1500 BC [7]. In 1866 Virchow reported several cases and introduced the term ochronosis to identify the condition characterized by pigmentation of the cartilages, the ligaments, tendons, and the intima of the large blood vessels [8]. This arthritis usually occurs in adult patients with Alkaptonuria in the third and fourth decade of life and is manifested with pain in the lower back, the thighs, and the large joints because of degenerative changes in the joint cartilages and the intervertebral discs $[9,10]$.

Alkaptonuric spondylosis can be detected along the whole spinal column but mostly affects the lumbar region [11].

The progressive course of the disease finally leads to ankylosing spondylitis because of severe degenerative discs and joints changes. But, in general, the severity of the radiological changes does not correspond with the complaints of the patients. For these reasons, the diagnosis of prolapsed intervertebral disc in ochronosis is unusual, and only a few cases were operated upon for the prolapsed disc [12].

Chanika Phornphutkul et al. studied the natural history of the disease and found the Life-table analyses showed that joint replacement was performed at a mean age of 55 years and that renal stones developed at 64 years, cardiac-valve involvement at 54 years, and coronary-artery calcification at 59 years. Linear regression analysis indicated that the radiographic score for the severity of disease began increasing after the age of 30 years, with a more rapid increase in men than in women [1].

The radiological findings were well described, and the progressive calcification and vacuum phenomenon of disc spaces are the most characteristic findings. Disc space narrowing is associated with calcification, and marginal sclerosis of the vertebral bodies is accentuated by osteopenia also. Progressive formation of marginal intervertebral bridges and obliteration of disc spaces at multiple levels ('pseudoblock vertebra') may occur. On MRI images, the striking feature is the multiple levels of disc prolapse, with the uniformly prominent low signal on $\mathrm{T} 2 \mathrm{~W}$ images in all the discs, suggesting generalized disc degeneration [13].

The earliest case report for a prolapsed lumbar disc in alkaptonuria patient was by Field et al. in 1963 [2], while Slowik et al. has described the only case of siblings with familial alkaptonuria presenting with lumbar disc disease [14]. Many authors reported surgically treated cases of the lumbar disc herniation in thoese group of patients with excellent outcome. In an article published in 2012, Prasad Krishnan, Siddhartha Roy Chowdhury reviewed all the published cases studies and found 14 cases [out of whom 1 patient presented with thoracic myelopathy due to disc prolapse and 1 with root canal stenosis [15]. Lumbar spinal stenosis in an alkaptonuria patient due to 'ligamentum flavum hypertrophy associated with ochronotic deposits' has been reported [16].

In the case presented in this paper the diagnosis was established retrospectively after the finding of the abnormal discoloration of the 
soft tissues and bones during performing the routine discectomy surgery which, in part, reflect the benign course of this congenital disease since the patient reached this age without a life threatening events related to the known cardiovascular complications associated with alkaptouria and that spondylotic arthropathy may be the first presentation particularly in the cases where the diagnosis was missed in early life.

Performing discectomy in such cases is associated with good outcome specially for the leg pain and claudication.

\section{Conclusion}

Alkaptonuria is a rare metabolic disease and the presentation with a prolapsed intervertebral disc prolapse requiring surgical intervention is even rarer. The lumbar region most prominently involved. This metabolic disorder is often recognized on physical examination following the surgery. The abnormal discoloration of the removed disc material durind surgery is the usual finding. Therefore, urinalysis for homogentisic acid should be performed in those patients. Surgical treatment for lumbar disc herniation in this group is successful.

\section{References}

1. Phornphutkul C, Introne WJ, Perry MB, Bernardini I, Murphey MD, et al. (2002) Natural history of alkaptonuria. N Engl J Med 347: 2111-2121.

2. Feild Jr, Higley GB Sr, Desaussure RL Jr (1963) Ochronosis with ruptured lumbar disc: case report. J Neurosurg 20: 348-351.

3. McCollum DE, Odom GL (1965) Alkaptonuria, ochronosis, and low-back pain. A case report. J Bone Joint Surg Am 47: 1389-1392.

4. Wirchov R (1866) On case of general ochronosis the cartilage and knorpela hnlichen parts. Arch Pathol Anat 37: 212.
5. Osler W (1904) Ochronosis: the pigmentation of cartilages, sclerotics and skin in alkaptonuria. Lancet 1:10.

6. Scriver CR (2000) The hyperphenylalaninemias and alkaptonuria. In: Goldmon L, Benett JC (eds.) Cecil text book of medicine. (21st edn), WB Saunders, Philadelphia, pp 1108-1110.

7. Stenn FF, Milgram JW, Lee SL, Weigand RJ, Veis A (1977) Biochemical identification of homogentisic acid pigment in an ochronotic egyptian mummy. Science 197: 566-568.

8. Khachadurian A, Feisal Ka (1958) Alkaptonuria; report of a family with seven cases appearing in four successive generations, with metabolic studies in one patient. J Chronic Dis 7: 455-465.

9. Laskar FH, Sargison KD (1970) Ochronotic arthropathy. A review with four case reports. J Bone Joint Surg Br 52: 653-666.

10. Kusakabe N, Tsuzuki N, Sonada M (1995) Compression of the cervical cord due to alcaptonuric arthropathy of the atlanto-axial joint. A case report. J Bone Joint Surg Am 77: 274-277.

11. Millea TP, Segal LS, Liss RG, Stauffer ES (1992) Spine fracture in ochronosis. Report of a case. Clin Orthop Relat Res : 208-211.

12. Reddy DR, Prasad VS (1998) Alkaptonuria presenting as lumbar disc prolapse: case report and review of literature. Spinal Cord 36: 523-524.

13. Bayindir P, Yilmaz Ovali G, Pabuayasu Y, Temiz C, Duruoz T (2006) Radiologic features of lumbar spine in ochronosis in late stages. Clin Rheumatol 25: 588-590.

14. Slowik J, Bandur M, Libuszowska D, Bereza M (1980) 2 cases of familial alkaptonuria with neurological complications. Neurol Neurochir Pol 14: 559-563.

15. Krishnan P, Chowdhury SR (2012) Lumbar disc herniation in a patient of alkaptonuria: Case report and review of literature Neurology. J neurology 60: 667-669.

16. Reddy R, Vijayasaradhi M, Biswal D (2012) Focal ligamentum flavum hypertrophy with ochronotic deposits: an unusual cause for neurogenic claudication in alkaptonuria. Asian Spine J 6:148-151. 